\title{
Prevalencia en México del tumor de células gigantes, osteosarcoma y condrosarcoma (2013-2017)
}

\author{
Prevalence in Mexico of giant cell tumor, osteosarcoma and chondrosarcoma (2013-2017) \\ Rivas-Berny C,* Méndez-Domínguez N,* Alejos-Gómez R ${ }^{\ddagger}$ \\ Escuela de Medicina. Universidad Marista de Mérida.
}

RESUMEN. Introducción: Los tumores óseos primarios constituyen $1 \%$ de los cánceres en el adulto, siendo los más frecuentes el osteosarcoma y el condrosarcoma. La tumoración de células gigantes (TCG) es una neoplasia benigna localmente agresiva, que representa de $4-10 \%$ de todos los tumores óseos primarios, pero en ciertas poblaciones representa el hasta $20 \%$. El objetivo principal de este trabajo fue determinar la proporción de los TCG, osteosarcomas y condrosarcomas en un período de cinco años y describir las características de las pacientes de los cuales proceden. Importancia clínica: se requieren más datos acerca de la epidemiología de los tumores de los huesos, para conocer su epidemiología en la población mexicana. Material y métodos: Estudio observacional descriptivo de corte transversal retrospectivo incluyendo a la totalidad de pacientes que fueron intervenidos quirúrgicamente mediante resección tumoral, en los hospitales públicos a nivel nacional, y cuyas piezas histopatológicas fueron procesadas y dieron como resultado tejido compatible con: a) TCG, b) osteosarcomas y c) condrosarcomas. Resultados: Entre el 2013 y 2017 se reportaron 138 casos de los tres tumores óseos primarios estudiados, el tumor de células gigantes (50\%), el osteosarcoma (25.36\%) y el condrosarcoma (24.64\%). Los estados que tuvieron mayor número de incidencias fueron el Estado de México (45.65\%) y la Ciudad de México (26.81\%). Las mujeres presentaron una mayor prevalencia (57.25\%) que
ABSTRACT. Introduction: Primary bone tumors make up $1 \%$ of cancers in adults, the most common being osteosarcoma and chondrosarcoma. Giant cell tumor (GCT) is a locally aggressive benign neoplasm, accounting for $4-10 \%$ of all primary bone tumors, but in certain populations it accounts for up to $20 \%$. The main objective of this work was to determine the proportion of GCTs, osteosarcomas and chondrosarcomas in a period of five years, and describe the characteristics of the patients from whom they come. Clinical implications: More information about the epidemiology of bone tumors is required to know their epidemiology in the Mexican population. Material and methods: Descriptive observational study of a retrospective cross section including all patients who underwent surgery for tumoral resection, in public hospitals at national level, and whose histopathological pieces were processed and resulted in tissue compatible with a) GCT, b) osteosarcomas and c) chondrosarcomas. Results: Between 2013 and 2017, 138 cases of the three primary bone tumors studied were reported, giant cell tumor (50\%), osteosarcoma (25.36\%) and chondrosarcoma (24.64\%). The states with the highest number of cases were the state of Mexico (45.65\%) and Mexico City (26.81\%). Women had a higher prevalence (57.25\%) than men $(42.75 \%)$. The average age of presentation of the tumors was 36.80 years. Conclusions: GCT is not an

\section{Nivel de evidencia: IV}

* Escuela de Medicina. Universidad Marista de Mérida. Mérida, Yucatán, México.

‡ Hospital General O’Horan.

Dirección para correspondencia:

Christian Rivas-Berny

Escuela de Medicina. Campus de Ciencias de la Salud. Universidad Marista de Mérida.

Periférico Norte Tablaje Catastral Núm. 13941,

Carretera Mérida-Progreso

C.P. 97300 , Mérida Yucatán, México.

Tel: (999) 2600-0094

E-mail: mionaitsir@gmail.com

Citar como: Rivas-Berny C, Méndez-Domínguez N, Alejos-Gómez R. Prevalencia en México del tumor de células gigantes, osteosarcoma y condrosarcoma (2013-2017). Acta Ortop Mex. 2020; 34(3): 183-188. https://dx.doi.org/10.35366/97071 
los hombres (42.75\%). La edad media de presentación de los tumores fue 36.80 años. Conclusiones: El TCG no es una tumoración infrecuente en la población mexicana, ya que cuenta con características propias.

Palabras clave: Tumor de células gigantes, osteosarcoma, condrosarcoma, prevalencia, México.

\section{Introducción}

Los tumores óseos primarios son un grupo de neoplasias benignas y malignas con un rango amplio de alteraciones genéticas y presentaciones histológicas. ${ }^{1}$ Constituyen $1 \%$ de los cánceres en el adulto, siendo el osteosarcoma el tumor más frecuente en todas las edades, seguido por el condrosarcoma en adultos. ${ }^{2}$

El tumor de células gigantes (TCG) es una neoplasia benigna localmente agresiva que se asocia con una gran variedad biológica, que varía desde una transformación maligna (10\%) hasta metástasis pulmonares (1-4\%), con tasas de recurrencia local que varían entre 10 y $75 \%$ de los pacientes. ${ }^{3,4,5}$ Representa de $4-10 \%$ de todos los tumores primarios de tipo óseo, pero en poblaciones como la india y china representa hasta $20 \%$, lo que hace plantearse la pregunta ¿cuál sería la prevalencia del TCG en la población mexicana?, esta neoplasia tiene una ligera predilección por las mujeres teniendo una relación hombre/mujer de 1:1.5.,5 La mayoría de los casos del TCG se presenta en personas entre los 20 y 40 años de edad y $<10 \%$ de las incidencias en población pediátrica y adultos mayores de 65 años. ${ }^{6}$ Usualmente involucra el final de un hueso largo. ${ }^{5}$ La mayoría se presenta, en uncommon tumor in the Mexican population, since it has its own characteristics.

Keywords: Giant cell tumor, osteosarcoma, chondrosarcoma, prevalence, Mexico.

orden decreciente, en las siguientes regiones óseas: fémur distal, tibia proximal, radio distal, sacro y húmero proximal, siendo la región de la rodilla la más frecuente (50\%), otros sitios de menor frecuencia son la cabeza del peroné y el fémur proximal. ${ }^{7}$

Debido a que la población mexicana cuenta con características demográficas propias que la diferencian de las poblaciones en las que se han realizado estudios acerca del TCG, y que en la actualidad hay pocos análisis de la prevalencia del TCG en población mexicana, llevó a que se planteara la siguiente hipótesis: la prevalencia del TCG es mayor en México que lo reportado en la literatura, derivando como objetivo de este trabajo determinar la proporción de los TCG, osteosarcomas y condrosarcomas en un período de cinco años y describir las características de las pacientes de las cuales proceden.

\section{Material y métodos}

Estudio observacional descriptivo de corte transversal retrospectivo, incluyendo a la totalidad de pacientes que fueron intervenidos quirúrgicamente para resección tumoral, en los hospitales públicos a nivel nacional, y cuyas piezas histopatológicas fueron procesadas y dieron

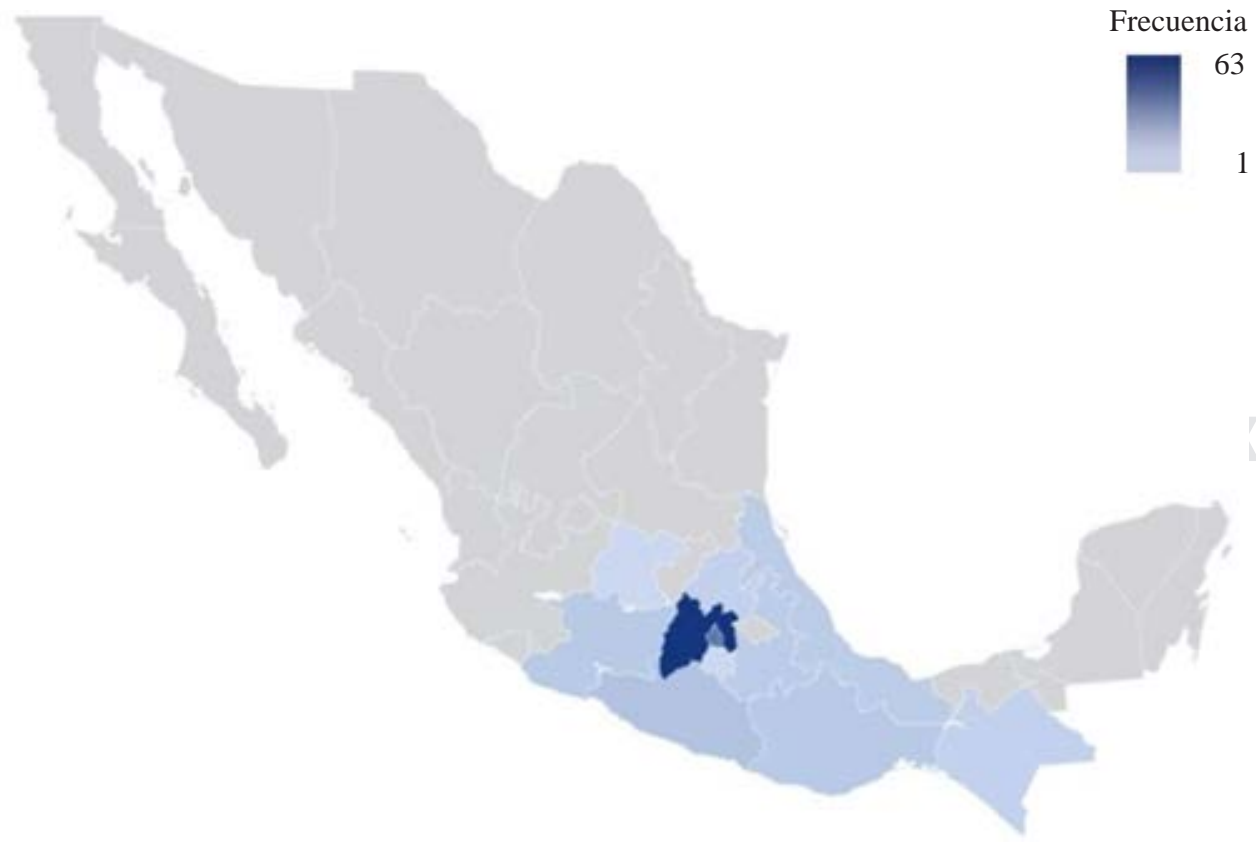

Figura 1:

Frecuencia del número de casos de los tumores óseos primarios, por estado, del 2013 al 2017. 
Tabla 1: Distribución del número de casos de los tumores óseos primarios, por unidad médica.

\begin{tabular}{|c|c|c|c|c|c|c|}
\hline \multirow[b]{2}{*}{ Unidad } & \multicolumn{2}{|c|}{ Tumor de células gigantes $n=69$} & \multicolumn{2}{|c|}{ Osteosarcoma $\mathrm{n}=35$} & \multicolumn{2}{|c|}{ Condrosarcoma $\mathrm{n}=34$} \\
\hline & $\mathrm{n}$ & (\%) & $\mathrm{n}$ & (\%) & $\mathrm{n}$ & (\%) \\
\hline Otorrinolaringología & 0 & $(0)$ & 1 & $(2.86)$ & 0 & (0) \\
\hline Cirugía Plástica & 12 & (17.39) & 3 & $(8.57)$ & 0 & $(0)$ \\
\hline Ortopedia & 25 & (36.23) & 3 & $(8.57)$ & 3 & (8.82) \\
\hline Oncología & 26 & (37.68) & 14 & $(40.00)$ & 28 & (82.35) \\
\hline Cirugía General & 0 & $(0)$ & 1 & $(2.86)$ & 0 & $(0)$ \\
\hline Neurología y neurocirugía & 1 & $(1.45)$ & 2 & $(5.71)$ & 1 & $(2.94)$ \\
\hline Estomatología & 1 & (1.45) & 0 & $(0)$ & 0 & $(0)$ \\
\hline Neumología & 1 & (1.45) & 0 & (0) & 1 & (2.94) \\
\hline Pediatría & 3 & (4.35) & 11 & (31.43) & 1 & (2.94) \\
\hline Total & 69 & $(100.00)$ & 35 & (100.00) & 34 & $(100.00)$ \\
\hline
\end{tabular}

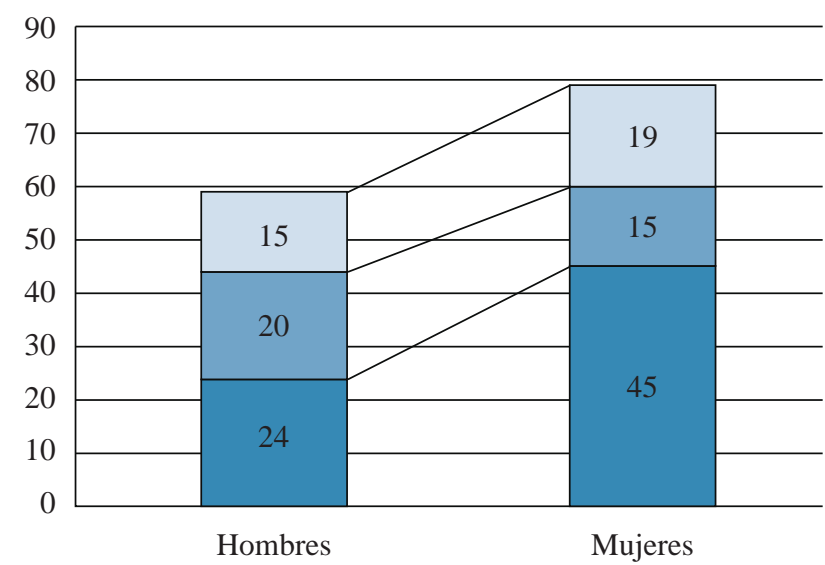

TCG $\square$ Osteosarcoma $\quad \square$ Condrosarcoma

Figura 2: Distribución del número de casos de los tumores óseos primarios, por sexo.

TCG = tumor de células gigantes

como resultado tejido compatible con: a) tumor de células gigantes, b) osteosarcomas y c) condrosarcomas, empleando la base de datos abierta de los registros de los resultados histopatológicos de las instituciones públicas de salud proporcionada por la Dirección General de Información de Salud (DGIS).

Se consideró como criterio de eliminación la ausencia de descripción histopatológica de la pieza recibida. Como variables se incluyeron, entre las sociodemográficas, la edad, sexo y lugar de residencia del paciente y como clínicas, el tipo de tejido tumoral, servicio médico de procedencia y región topográfica de localización en el cuerpo.

La edad se analizó como variable continua, pero también como variable nominal en el rango infantil (de cero a 10 años), adolescentes (de 11 a 19 años), adultos jóvenes (de 20 a 39 años), adultos maduros (de 40-59 años) y adultos mayores (60 y más).

Las ubicaciones anatómicas de las lesiones óseas del TCG y el osteosarcoma se clasificaron, para los fines de este estudio, de acuerdo a su distribución en: esquelética (axial/

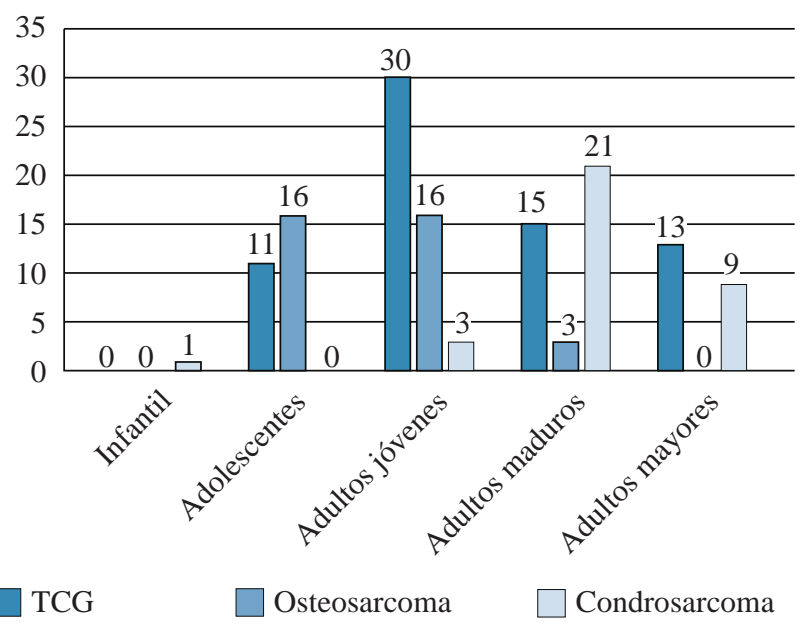

Figura 3: Distribución del número de casos de los tumores óseos primarios, por grupo de edad.

TCG = tumor de células gigantes.

apendicular), de extremidad (superior/inferior) y el hueso o región anatómica afectada.

Métodos estadísticos: los datos se analizaron mediante estadística descriptiva, obteniendo los totales, medidas de tendencia central y dispersión. Para la comparación entre grupos por edad, sexo, residencia, tipo de tejido tumoral y servicio médico de referencia, se realizaron pruebas de comparación de medias (t Student) y de proporciones $\left(\chi^{2}\right)$, estableciendo los intervalos de confianza al $95 \%$ y error máximo de 5\%. Se generaron tablas, gráficas y esquemas de representación en mapas e ilustraciones anatómicas. La estadística descriptiva y de contraste de hipótesis se generó empleando el programa Stata $14^{\circledR}$.

Resultados

En los años comprendidos entre el 2013 y 2017 se reportaron 138 casos de los tres tumores óseos primarios incluidos en el estudio, el TCG obtuvo la prevalencia más 
alta 50\% ( $n=69)$, seguido por el osteosarcoma 25.36\% ( $\mathrm{n}=$ $35)$ y el condrosarcoma $24.64 \%(n=34)$.

La prevalencia general de los tumores óseos primarios, analizados según el estado de procedencia, fue más alta en el Estado de México 45.65\% ( $=63)$, seguido por la Ciudad de México 26.81\% ( $n=37)$, X 5.8\% ( $n=8)$, Oaxaca 4.35\% $(\mathrm{n}=6)$, Michoacán 4.35\% ( $\mathrm{n}=6)$, no se mencionaron los estados que tuvieron menos de seis casos (Figura 1).

De los 138 eventos estudiados, las mujeres presentaron $57.25 \%(n=79)$ y los hombres $42.75 \%(n=59)$. Particularmente, en los tumores de células gigantes, se demostró una mayor proporción de incidencias en féminas, representando $65.22 \%$ de los casos (45 casos) y los hombres $34.78 \%$ (24 casos) respectivamente, con una relación hombre/mujer 0.35:0.65 casos. En relación al osteosarcoma, los varones fueron más afectados con $57.14 \%(n=20)$, que las mujeres $42.86 \%(n=15)$, una relación 0.57:0.43 casos. En el caso del condrosarcoma la distribución fue la siguiente: 44.12 y 55.88\% hombres y mujeres respectivamente, con una relación 0.44:0.56 casos, como se muestra en la Figura 2.

En el TCG, la edad media de presentación en los hombres fue 36.21 años y en las mujeres 37.37 años ( $p=0.694)$, en el caso del osteosarcoma, en los varones fue 22.28 años y en las féminas 41.72 años $(p=0.000)$ y en el condrosarcoma, para los hombres fue 52.91 años y en las mujeres 31.52 años $(p=0.000)$. La distribución por grupos de edad de los tumores óseos primarios fue la siguiente, infantil: 0.72\% $(\mathrm{n}=1)$; adolescentes: $19.57 \%(\mathrm{n}=27)$; adultos jóvenes: 35.51\% ( $n=49)$; adultos maduros: $28.26 \%(n=39)$; adultos mayores: $15.94 \%(n=22)$. En la Figura 3 se muestra el número de casos por grupos de edad de los tumores óseos primarios de forma individual.

Las unidades médicas en las que fueron atendidos tales tumores de los huesos se representan en la Tabla 1.

La distribución anatómica de las lesiones óseas del TCG fue la siguiente (Figura 4): esqueleto axial; 1.45\% (n = 1); esqueleto apendicular: 81.16\% $(\mathrm{n}=56)$; extremidad superior: 43.48\% ( $n=30)$; extremidad inferior: 30.43\% $(n=21)$; mano $33.33 \%(n=33)$, no se mencionaron los huesos o regiones que en las que se reportó un número de incidentes menor a 30. La distribución en el osteosarcoma fue (Figura 5): esqueleto axial: $22.86 \%(n=8)$; esqueleto apendicular: $51.43 \%(n=$ 18); extremidad superior: $5.71 \%$ (dos casos); extremidad inferior: 45.71\% ( $n=16)$; mandíbula: 14.29\% $(n=5)$; tibia: $8.57 \%(n=3)$, no se mencionaron los huesos o regiones que en las que se reportó un número de casos menor a tres.

\section{Discusión}

En el presente estudio se confirma que, en la población mexicana, tuvo una mayor prevalencia el TCG que los otros

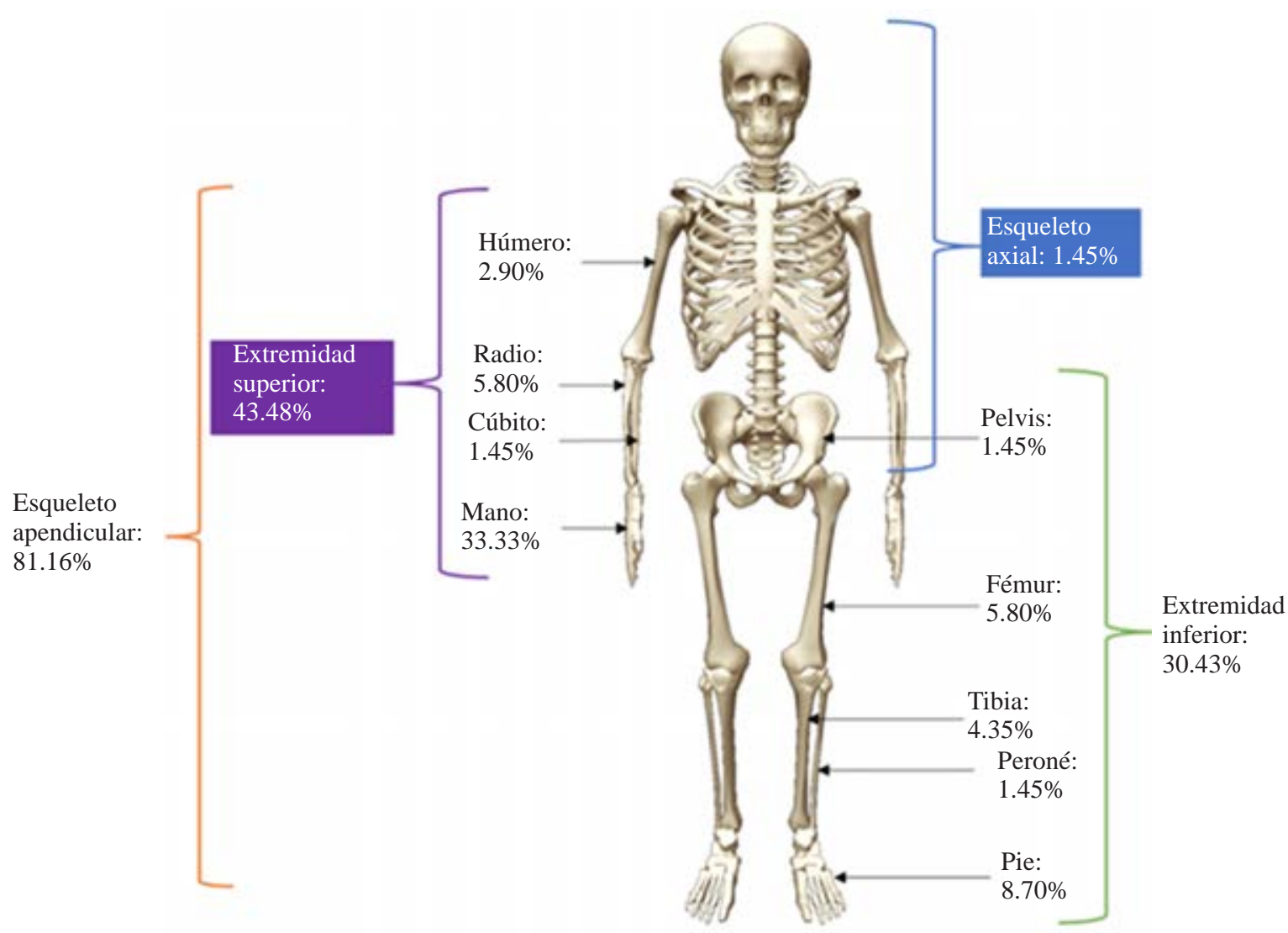

Esqueleto sin clasificar: $17.39 \%$

Extremidad sin clasificar: $26.09 \%$

Huesos sin clasificar: $34.78 \%$ 


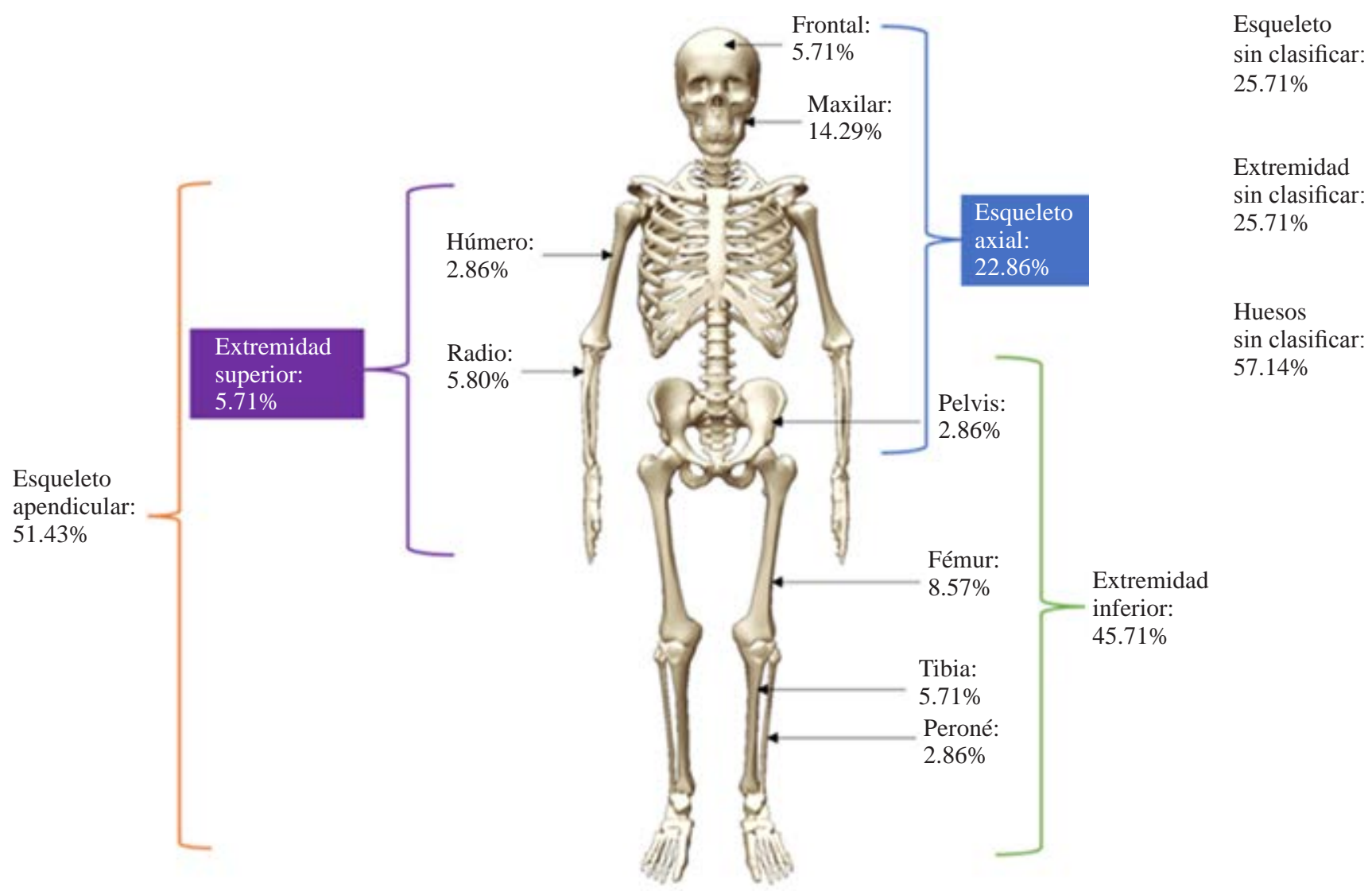

Figura 5: Distribución anatómica de las lesiones del osteosarcoma.

tumores incluidos en el estudio en los años comprendidos entre el 2013-2017.

La distribución de la prevalencia de los tumores óseos primarios (tumor de células gigantes, osteosarcoma y condrosarcoma) en población mexicana fue idéntica a la obtenida por De la Garza-Montano y colaboradores, ${ }^{8}$ siendo el TCG el más representativo de los tres tumores. La distribución geográfica de los tumores óseos primarios resultó ser similar a la que obtuvieron De la Garza-Montano y colaboradores ${ }^{8}$ en su investigación, y en el caso de la distribución geográfica sólo del TCG resultó ser similar a la que obtuvieron Estrada-Villaseñor y colaboradores, ${ }^{9}$ con lo que se puede inferir la posible existencia de factores ambientales en la zona centro-sur de México que pudieran estar condicionando la aparición de este tipo de tumores, teniendo como limitante la ausencia de estudios de prevalencia en México de los tumores óseos primarios, para poder vislumbrar un panorama epidemiológico completo, ya que en un mismo país puede haber diferencias en la prevalencia de la población urbana y rural, como en la investigación realizada en Suecia por Justyna y colaboradores. $^{5}$

Varios trabajos han informado que el TCG ocurre predominantemente en mujeres, $3,5,6,7,7,8,10,11,12$ el presente estudio no fue la excepción, siendo las féminas las más afectadas por el TCG; el osteosarcoma, como se menciona en la literatura, $, 12,13,14$ es más frecuente en los hombres y en el caso del condrosarcoma, en investigación realizada por De la Garza-Montano y colaboradores, ${ }^{8}$ fue más frecuente en las mujeres, pero en el efectuado por Bindiganavile y colaboradores ${ }^{15}$ fue más frecuente en los varones, por lo que se revela que hacen falta más estudios acerca de la demografía del condrosarcoma para obtener información confiable sobre distribución de frecuencia en hombres y mujeres.

En este estudio, las edades promedio de los tumores fueron similares a las que obtuvieron De la GarzaMontano y colaboradores, ${ }^{8}$ en la que las edades medias de presentación de dichos tumores fue 34.45 años para el TCG, 24.3 y 49.22 años para el osteosarcoma y el condrosarcoma respectivamente. En cuanto a los resultados del número de casos por grupo etario en el TCG fueron similares a los obtenidos por Estrada-Villaseñor y colaboradores. ${ }^{9}$

En ningún otro artículo de la literatura revisada se encontró la unidad de procedencia de los tumores óseos primarios, por lo cual ha de considerarse incluirla en el estudio ya que es importante conocer que unidades son las que están atendiendo estos tumores, a fin de promover una derivación temprana a la unidad correspondiente. Con respecto a localización del TCG, fue más frecuente en el esqueleto apendicular como en el ensayo realizado por Justyna y colaboradores, ${ }^{5}$ con la diferencia de que en 
su trabajo la extremidad inferior era donde se encontraba la mayoría de los casos. En ninguno de los otros estudios de TCG hubo coincidencia con la región anatómica más frecuentemente afectada, siendo la mano la más frecuente en el presente trabajo.

Debido a que la base de datos utilizada para el estudio no menciona si el caso fue una recidiva o no, se puede inferir que algunos de los eventos de TCG incluidos pueden corresponder a recidivas, ya que en el TCG, a pesar de ser una neoplasia benigna, existe un riesgo de recidiva local que se observa en $10-40 \%$ de los casos, ${ }^{6}$ por lo cual es importante conocer los tratamientos adyuvantes en el legrado que reducen el riesgo de recidiva local. Entre las opciones utilizadas se encuentran: fresado, polimetilmetacrilato, nitrógeno, etanol, fenol, entre otros. ${ }^{6,16}$ El polimetilmetacrilato es el adyuvante más utilizado y ha demostrado reducir el riesgo de recurrencia local de 49$22 \%{ }^{16,17}$ Aunque existe evidencia que sugiere que el haber realizado una escisión meticulosa mediante un legrado no requiere la adición de un tratamiento adyuvante. ${ }^{6}$

El presente estudio, al ser de diseño transversal, adolece de ciertas limitaciones que deberían tomarse en cuenta al momento de interpretar los resultados del estudio, como son su corte retrospectivo y la dependencia de datos codificados que están sujetos al error humano al momento de su codificación. Dichas limitantes podrían haber llevado a una subestimación del tamaño del problema. Se espera que este estudio sea motivo de nuevas investigaciones acerca los tumores óseos primarios en México, ya que al ser un país de con una gran diversidad poblacional y sabiendo que el TCG puede comportarse de una forma diferente entre los distintos sectores de la población, es necesario conocer el sector más afectado y así poder atender sus necesidades.

\section{Conclusión}

Los datos, obtenidos de la base de datos abierta de los registros de los resultados histopatológicos, revelaron que el TCG no es una tumoración infrecuente en la población mexicana, pero es necesaria la realización de más trabajos sobre el TCG en el norte y sureste de México para poder conocer la epidemiología del TCG en México.

Bibliografía

1. Flanagan A, Lindsay D. A diagnostic approach to bone tumours. Pathology. 2017; 49(7): 675-87. doi: 10.1016/j.pathol.2017.08.003.
2. Kumar N, Gupta B. Global incidence of primary malignant bone tumors. Curr Orthop Pract. 2016; 27(5): 530-4. doi: 10.1097/ BCO.0000000000000405.

3. Mavrogenis A, Igoumenou V, Megaloikonomos P, Panagopoulos G, Papagelopoulos P, Soucacos P. Giant cell tumor of bone revisited. SICOT J. 2017; 3: 54. doi: 10.1051/sicotj/2017041.

4. Lin F, Hu Y, Zhao L, Zhang H, Yu X, Wang Z, et al. The epidemiological and clinical features of primary giant cell tumor around the knee: a report from the multicenter retrospective study in china. $J$ Bone Oncol. 2016; 5(1): 38-42. doi: 10.1016/j.jbo.2016.02.001.

5. Amelio JM, Rockberg J, Hernandez RK, Sobocki P, Stryker S, Bach BA, et al. Population-based study of giant cell tumor of bone in Sweden (1983-2011). Cancer Epidemiol. 2016; 42: 82-89. doi: 0.1016/j.canep.2016.03.014.

6. Gouin F, Odri G, Revert R, Heymann MF, Rédini F. Tumores de células gigantes de los huesos. EMC-Aparato Locomotor. 2012; 45(2): 1-13. doi: 10.1016/s1286-935x(12)61891-7.

7. Raskin K, Schwab J, Mankin H, Springfield D, Hornicek F. Giant Cell Tumor of Bone. J Am Acad Orthop Surg. 2013; 21(2): 118-26. doi: 10.5435/JAAOS-21-02-118.

8. Sobti A, Agrawal P, Agarwala S, Agarwal M. Giant Cell Tumor of Bone - An Overview. Arch Bone Jt Surg. 2016; 4(1): 2-9. doi: 10.22038/ABJS.2016.4701.

9. De la Garza-Montano P, Estrada-Villasenor E, Rubio RD, MartinezLopez V, Avila-Luna A, Alfaro-Rodriguez A, et al. Epidemiological aspects of osteosarcoma, giant cell tumor and chondrosarcoma musculoskeletal tumors. Experience of the National Rehabilitation Institute, Mexico City. Asian Pac J Cancer Prev. 2015; 16(15): 64515. doi: 10.7314/apjcp.2015.16.15.6451.

10. Estrada-Villaseñor E, Linares-González L, Delgado-Cedillo E, González-Guzmán R, Rico-Martínez G. Prevalencia y características clínico-patológicas del tumor de células gigantes. Acta Ortop. Mex. 2015; 29(6): 295-8. doi: n/a.

11. Verschoor AJ, Bovée JVMG, Mastboom MJL, Dijkstra PDS, Michiel AJ Van De Sande, Gelderblom H. Incidence and demographics of giant cell tumor of bone in The Netherlands: first nationwide pathology registry study. Acta Orthop. 2018; 89(5): 570-4. doi: 10.1080/17453674.2018.1490987.

12. Rockberg J, Bach BA, Amelio J, Hernandez RK, Sobocki P, Engellau $\mathrm{J}$, et al. Incidence Trends in the Diagnosis of Giant Cell Tumor of Bone in Sweden Since 1958. J Bone Joint Surg Am. 2015; 97(21): 1756-66. doi: 10.2106/JBJS.O.00156.

13. Geller DS, Gorlick R. Osteosarcoma: a review of diagnosis, management, and treatment strategies. Clin Adv Hematol Oncol. 2010; 8(10): 705-18. doi: n/a.

14. López P, Álvarez S, González JL. Actualización del osteosarcoma para el médico de familia. SEMERGEN-Medicina de Familia. 2011; 37(1): 1-52. doi: 10.1016/j.semerg.2010.06.008.

15. Bindiganavile S, Han I, Yun JY, Kim H-S. Long-term outcome of chondrosarcoma: a single institutional experience. Cancer Res Treat. 2015; 47(4): 897-903. doi: 10.4143/crt.2014.135.

16. Klenke FM, Wenger DE, Inwards CY, Rose PS, Sim FH. Giant cell tumor of bone: risk factors for recurrence. Clin Orthop Relat Res. 2011; 469(2): 591-9. doi: 10.1007/s11999-010-1501-7.

17. Gouin F, Dumaine V; French Sarcoma and Bone Tumor Study Groups GSF-GETO. Local recurrence after curettage treatment of giant cell tumors in peripheral bones: retrospective study by the GSF-GETO (French Sarcoma and Bone Tumor Study Groups). Orthop Traumatol Surg Res. 2013; 99(6 Suppl): S313-8. doi: 10.1016/j.otsr.2013.07.006. 